\title{
CONTRIBUTION OF MAGNETIC RESONANCE TO THE DIAGNOSIS OF CReutzFeldT-Jakob diseAse
}

\author{
CONTRIBUIÇÃO DA RESSONÂNCIA MAGNÉTICA NO DIAGNÓSTICO \\ DA DOENÇA DE CREUTZFELDT-JAKOB
}

\section{Leonardo Cordenonzi Pedroso de Albuquerque ${ }^{1}$, Juliana Avila Duarte ${ }^{2}$, Artur Francisco Schumacher Schuh ${ }^{1}$}

Clin Biomed Res. 2015;35(4):246-247

1 Serviço de Neurologia, Hospital de Clínicas de Porto Alegre. Porto Alegre, RS, Brazil.

2 Serviço de Radiologia, Hospital de Clínicas de Porto Alegre. Porto Alegre, RS, Brazil.

Corresponding author: Juliana Avila Duarte

E-mail: juavila2003@yahoo.com.br Serviço de Radiologia

Hospital de Clínicas de Porto Alegre Rua Ramiro Barcelos, 2350. 90035-903, Porto Alegre, RS, Brazil.
A71 year-old woman presented to our hospital with acute onset of progressive cognitive impairment and psychotic symptoms. Five weeks earlier, she noticed memory impairment and diplopia. After 2 weeks, she was admitted to a psychiatric hospital due to delusions, agitation and hallucinations. After 3 weeks, she was referred to our hospital with progressive deterioration, especially impairment of language and gait. At admission, examination showed aphasia, myoclonic jerks, apraxic gait, and Babinski's sign. No other neurological signs were noticed, and general medical examination was unremarkable. She had no previous psychiatric or significant medical conditions.

Laboratory tests showed mild elevation in anti-thyroid peroxidase titer. Other tests were normal, including complete blood count and tests for liver and renal function, electrolytes, TSH, vitamin B12, inflammatory markers, and HIV and syphilis antibody. Electroencephalogram (EEG) showed a semi-periodic pattern of widespread sharp-wave discharges with periods of arrhythmic widespread theta waves. FLAIR brain magnetic resonance imaging (MRI) (figure 1) showed hyperintensity and decreased diffusivity in the basal ganglia, specially in the head of caudate and putamen, as well as in the cortex of frontal lobes bilaterally, left parietal lobe and bilateral occipital lobes on diffusion weighted images (DWI) (figure 2) and apparent diffusion map. There was no gadolinium enhancement. All of these findings led to diagnosis of probable Creutzfeldt-Jakob disease (CJD) ${ }^{1}$.

CJD is a fatal prion-related neurodegenerative disease. This condition is categorized into sporadic CJD (sCJD), familial CJD (fCJD) and variant CJD (VCJD) ${ }^{2}$. SCJD is the most common variant, accounting for $85-90 \%$ of the cases, with an incidence of 1-1.5 per million annually ${ }^{1}$. fCJD is related to mutations in the prion protein gene (PRNP) on chromosome 20, and accounts for $10-15 \%$ of the cases. VCJD is related to bovine spongiform encephalopathy and accounts for $1 \%$ of the cases ${ }^{1,2}$.

CJD primarily presented with cognitive impairment, behavioral symptoms, and sensory and motor dysfunction. SCJD often presents with dementia, generally associated with ataxia and myoclonus, while VCJD has prominent behavioral symptoms, and may be misdiagnosed as a psychiatric illness. These diseases are rapidly progressive, with median survival of 5 months for SCJD, while VCJD has a slightly protracted course ${ }^{2,3}$.

The definite diagnosis requires pathological confirmation by biopsy. The cerebrospinal fluid (CSF) can be examined for biomarkers, such the 14-3-3 protein, but its use is controversial for high sensitivity and low speficity ${ }^{1}$. EEG shows a pattern with periodic sharp and slow wave complexes, with sensitivity of $65 \%$ and specificity of $80 \%{ }^{1}$. FLAIR and DWI MRI show hyperintensities in the cortex, putamen and caudate nucleus, providing a sensitivity and specificity of more than $90 \%{ }^{2}$. 
In a recent study comparing MRI and CSF biomarkers, MRI had a $97 \%$ accuracy in predicting the CJD diagnosis, versus $79.6 \%$ of total tau protein (T-tau), $70.4 \%$ of $14-3-3$ protein, and $71.4 \%$ of

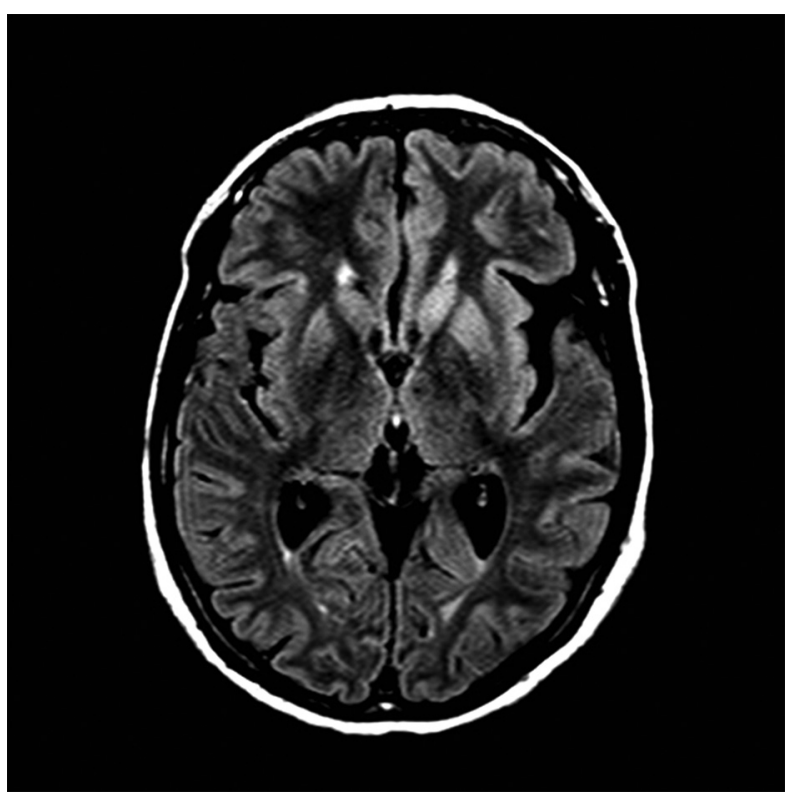

Figure 1: Axial flair weighted image - Frontal, temporal and occipital lobes showing discrete cortical and basal ganglia hyperintensities. neuron specific enolase ${ }^{4}$, and is currently the best non-invasive test for CJD diagnosis².

Unfortunately, the disease is untreatable, invariably progressive, and fatal in $100 \%$ of the cases.

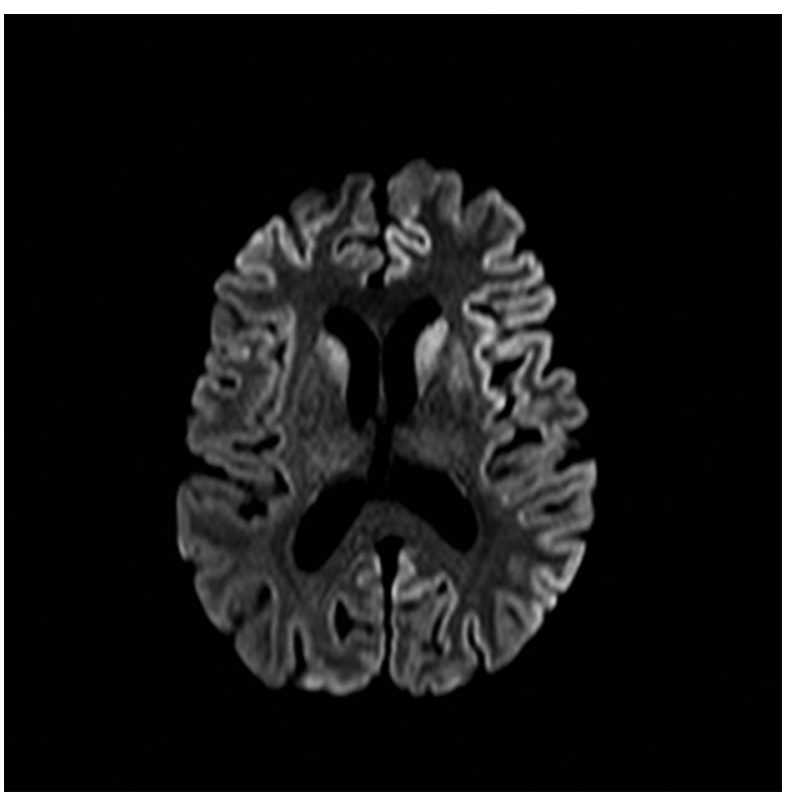

Figure 2: Diffusion weighted image and apparent diffusion map (not shown here) revealing restricted diffusion on the frontal, temporal and occipital lobes, strongly suggestive of Creutzfeldt Jacob disease.

\section{REFERENCES}

1. Lee J, Hyeon JW, Kim SY, Hwang KJ, Ju YR, Ryou C. Review: laboratory diagnosis and surveillance of

Creutzfeldt-Jakob disease. J Med Virol. 2015;87(1):175-86.
2. Rosenbloom MH, Atri A. The evaluation of rapidly progressive dementia. Neurologist. 2011;17(2):67-74.

3. Brown K, Mastrianni JA. The prion diseases. J Geriatr Psychiatry Neurol. 2010;23(4):277-98.
4. Forner SA, Takada LT, Bettcher BM, Lobach IV, Tartaglia MC, Torres-Chae $\mathrm{C}$, et al. Comparing CSF biomarkers and brain MRI in the diagnosis of sporadic Creutzfeldt-Jakob disease. Neurol Clin Pract. 2015;5(2):116-25.

Received: Sept 30, 2015 Accepted: Nov 11, 2015 